\title{
La enseñanza de la Arquitectura
}

Pier Luigi Nervi

Traducción de Gustavo Carabajal

Publicado en A\&P Continuidad \#03, diciembre de 2015
S bien se discute mucho de Arquitectura en todos los ambientes culturales y artísticos, creo que no se considera ni profundiza suficientemente la cuestión de las relaciones sustanciales y formales que relacionan los problemas arquitectónicos con la técnica constructiva.

En otras palabras, creo que no se pone suficientemente en evidencia el lugar que le corresponde, en la más íntima esencia de la actual situación arquitectónica, al desarrollo de las teorías estáticas y a los métodos constructivos consecuentes con el progreso científico y técnico de nuestra época en general.

Es evidente que la técnica por sí sola no puede dar espíritu y vida a verdaderas expresiones arquitectónicas, y que éstas no podrían adquirir forma sin una técnica realizadora pero, más allá de esta inmediata dependencia entre ideación y realización -válida para todos los tiempos y todas las técnicas dirigidas a dar cuerpo sensible a una idea abstracta-, se puede observar que en el campo arquitectónico sucedió algo mucho más importante, solo comparable a la transformación que sufrió la música luego de la invención de su escritura y de los instrumentos que forman la orquesta.

Antiguamente, el arquitecto, imposibilitado de realizar cálculos estáticos de verificación -ni siquiera aproximativos- se veía obligado a adoptar sistemas constructivos encontrados intuitivamente, perfeccionados sucesivamente a partir de un lento progreso empírico. El pasaje de un esquema estático-constructivo a otro, por ejemplo, de la estructura arquitrabada al uso de arco, se realiza lentamente por grados sucesivos. Definido un esquema, el mismo se mantenía vigente por un notable número de décadas o siglos hasta que surgía una nueva intuición constructiva.

Las posibilidades de las cuales hoy disponemos, para investigar cuantitativa y cualitativamente el comportamiento estático de cualquier disposición estructural (cuando sea insuficiente la verificación teóricomatemática está siempre abierta la opción de operar sobre modelos experimentales) y la eficiencia de los nuevos materiales constructivos, nos han brindado -en pocas decenas de años- una casi completa libertad de invención de nuevos esquemas constructivos, los cuales han anulado prácticamente todos los precedentes.

¿Quién pensaría hoy construir un estadio en mampostería y piedra de corte como el Coliseo o una cúpula de cinco metros de espesor como la de Santa María del Fiore en Florencia? a libertad de invención alcanzada, la mayor eficiencia estática de los nuevos materiales y la novedad de un gran número de temas constructivos, sin precedentes en la historia de la humanidad (estaciones ferroviarias, marítimas, aéreas, grandes talleres, centrales eléctricas, autopistas y grandes puentes necesarios al tráfico veloz), han modificado tan profundamente el construir de hoy que se hace no solo ilógico sino prácticamente imposible cualquier sustancial referencia a las obras del pasado, la cual -en todo caso- se limitaría a elementos singulares de carácter puramente formal. Es inútil insistir sobre lo absurdo y artificial que resulta la inserción de elementos pertenecientes a técnicas y arquitecturas del pasado en estructuras actuales, tan absurdo como resultaría la aplicación del castillo de popa o el mascarón de proa de los buques de seiscientos a un transatlántico moderno. El más ávido misoneísmo no podría detener la revolución arquitectónica, hoy en acto, como no podría revivir los grandes veleros o los viajes en diligencia.

La esencia del problema arquitectónico actual no es la búsqueda de imposibles conexiones con el pasado, sino más bien el aprovechamiento pleno -con espíritu libre- de las posibilidades constructivas que el progreso técnico nos ha dado.

Es necesario -sobre todo- dar alma y expresión estética a las nuevas técnicas edilicias, desarrollando a pleno la ilimitada riqueza. Para lograrlo es indispensable que el arquitecto, entendido como creador de la obra arquitectónica, posea un completo dominio de la técnica misma conociendo sus posibilidades y límites. ¿Cómo podría, de otro modo, expresar un pensamiento poético en un idioma del cual mal conozca palabras, gramática y sintaxis? Gran parte de las dificultades de gramática y sintaxis? Gran parte de las dificultades de
actual momento arquitectónico derivan precisamente del hecho que la rapidez del progreso técnico ha sobrepasado demasiado la inevitable lentitud de desarrollo de la preparación técnica de los proyectistas. Dado que esta preparación encuentra su insustituible base en los estudios universitarios, se puede afirmar que uno de los más importantes y sustanciales problemas -entre todos aquéllos que se presentan hoy en campo arquitectónico- es aquél de un eficaz programa de estudios de las facultades de Arquitectura.

No sabría cómo recomendar a todos aquéllos que aman la Arquitectura y se preocupan por su futuro, de poner en primer plano y considerar en toda su dimensión e importancia la cuestión de los planes de estudio de las escuelas superiores de Arquitectura.

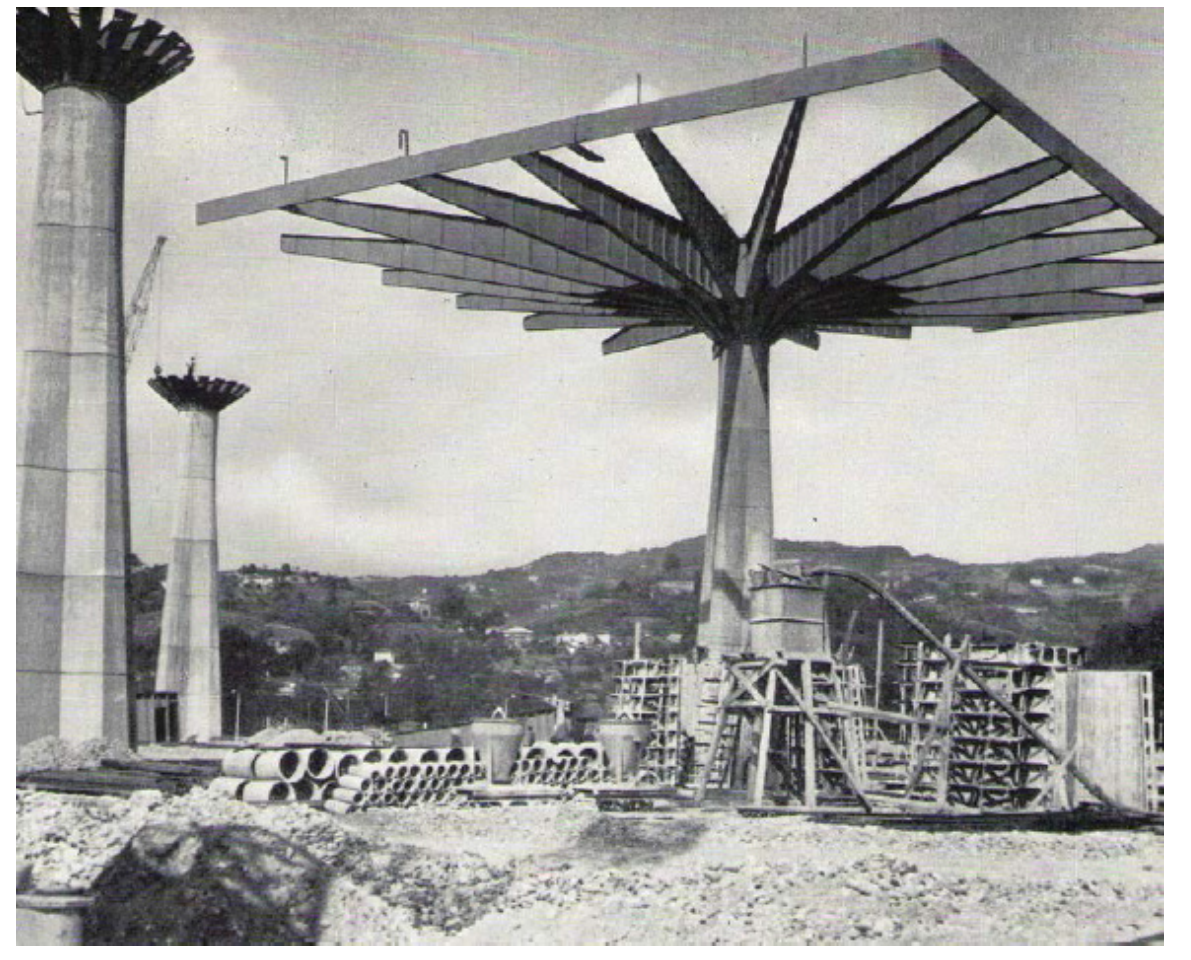

Se debería orientar a las escuelas de Arquitectura a la formación de verdaderos creadores del fenómeno arquitectónico que adquiere forma a través de la armoniosa fusión de todas las técnicas.

a esencia del problema está en la claridad de los métodos de enseñanza idóneos para brindar a los jóvenes arquitectos el dominio de la estática y de todas las técnicas subsidiarias de una construcción moderna (calefacción, acondicionamiento del aire, iluminación) sin sobrecargar excesivamente los ya pesados programas ni volverlos áridos envolviéndolos en un tecnicismo frío - a base de matemática- como sucede en las escuelas de Ingeniería. Se trata de que el arquitecto sea capaz de inventar una estructura compleja comprendiendo las posibilidades resistentes sin obligarlo a pasar por el desarrollo de la más elevada teoría de los sistemas elásticos, teoría vasta y compleja de por sí capaz de absorber todas las fuerzas de una inteligencia elevada. Considero que un buen resultado se podría obtener intentando hacer que la enseñanza de las distintas disciplinas técnicas, y de aquéllas propedéuticas relacionadas con estas últimas, sea lo más conceptual posible.

El arquitecto no necesita fórmulas exactas para calcular en detalle, sino conceptos bien claros en sentido cualitativo y ampliamente aproximativos en sentido cuantitativo. Para inventar y proporcionar una estructura, con aquella aproximación suficiente para definir el funcionamiento estático y las proporciones estáticas, no son necesarios cálculos exactos; éstos podrán ser realizados por los especialistas -en un segundo momento- en el desarrollo ejecutivo.

Cualquier estructura, inclusive la más complejas, se presta -si se la comprende y siente profundamentea verificaciones estáticas simples y rápidas que no necesitan que se recurra a desarrollos matemáticos siempre engorrosos- inútilmente polarizantes que pueden llegar a ser inclusive contraproducentes en la delica-da fase -fundamentalmente inventiva- del anteproyecto. Considero, por lo tanto, que el estudio de la estática y de la ciencia de las construcciones debería ser conducido, en las escuelas de Arquitectura, en manera completamente distinta de las escuelas de Ingeniería. Diversidad que no debe significar superficialidad, más bien profundidad intuitiva, comprensión íntima y capacidad de síntesis, en fórmulas simples para la primera aproximación a los procedimientos matemáticos de cálculo.

La fotoelasticidad, y la experimentación con extensómetros sobre modelos de estructura, podrían cooperar muy eficientemente en la comprensión del funcionamiento estático de las estructuras portantes. También la estática gráfica, con su representación visiva de las fuerzas, su manera de componerse y equilibrarse, debería ser desarrollada ampliamente.

Considero que el mismo método, dirigido a brindar una comprensión fundamentalmente cualitativa y conceptual, podría ser aplicado a las técnicas accesorias (calefacción, ventilación, iluminación) tan importantes en el resultado de una obra edilicia. En una palabra, se debería orientar a las escuelas de Arquitectura a la formación de verdaderos creadores del fenómeno arquitectónico que adquiere forma a través de la armoniosa fusión de todas las técnicas, resultado difícil de alcanzar si el arquitecto no conoce en profundidad los modos, los límites y las posibilidades de cada rama de la técnica y no se encuentra apto para coordinar y guiar el trabajo de los especialistas, cada uno de los cuales debe superar en fertilidad de intuición y claridad de concepto. 